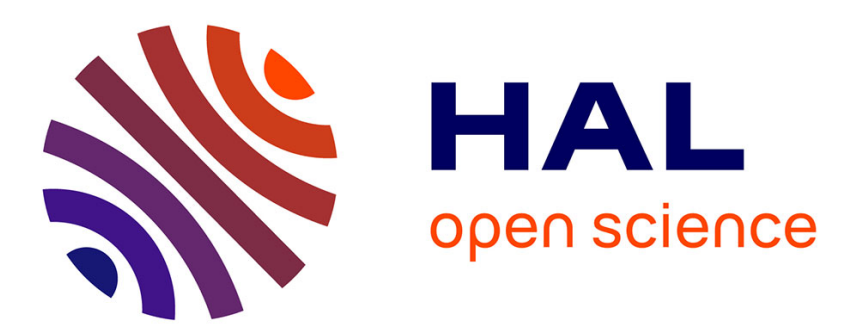

\title{
An undergraduate experiment to illustrate spatial transfer function concepts in Fourier optics
}

Jérôme Salvi, Gil Fanjoux, Anne Boetsch, Remo Giust

\section{To cite this version:}

Jérôme Salvi, Gil Fanjoux, Anne Boetsch, Remo Giust. An undergraduate experiment to illustrate spatial transfer function concepts in Fourier optics. American Journal of Physics, 2020, 88 (8), pp.617

- 624. hal-03360765

\section{HAL Id: hal-03360765 \\ https://hal.science/hal-03360765}

Submitted on 1 Oct 2021

HAL is a multi-disciplinary open access archive for the deposit and dissemination of scientific research documents, whether they are published or not. The documents may come from teaching and research institutions in France or abroad, or from public or private research centers.
L'archive ouverte pluridisciplinaire HAL, est destinée au dépôt et à la diffusion de documents scientifiques de niveau recherche, publiés ou non, émanant des établissements d'enseignement et de recherche français ou étrangers, des laboratoires publics ou privés. 


\title{
An undergraduate experiment to illustrate spatial transfer function concepts in Fourier optics
}

\author{
Jérôme Salvi,* Gil Fanjoux, Anne Boetsch, and Remo Giust
}

(Dated: May 13, 2020)

\begin{abstract}
Fourier analysis is a key tool in physics and engineering in the broadest sense, and is particularly used in the field of optics to design and study the properties of advanced imaging systems. Fourier optics as a distinct topic is usually taught in final-year undergraduate or first-year postgraduate studies, and a wide range of teaching approaches have been used to describe and explain its key concepts. Concerning practical work to accompany classroom lectures, the simplest laboratory experiments are based on studying how the properties of some diffracting aperture (pupil) is related to the corresponding Fraunhofer diffraction pattern, but this approach usually does not consider in detail the concepts of the optical transfer function and spatial frequencies. We describe here a simple experimental set-up that fills this particular gap and that illustrates the spatial frequency-domain response of a simple optical system using incoherent light. The Modulation Transfer Function is directly measured for several different pupil geometries, and shows very good agreement with theoretical calculations. This experiment clearly demonstrates spatial transfer function concepts in Fourier optics, complementing and extending other studies of Fourier transforms in physics that may consider similar ideas in a time and frequency signal-processing context.
\end{abstract}




\section{INTRODUCTION}

With the rapidly increasing importance of advanced imaging techniques in physics and technology, Fourier optics is widely-recognized as essential background for any optics engineer or student in physics. The basic concepts of Fourier optics applied to imaging date back to the 1940s, first introduced by Duffieux ${ }^{1,2}$ and then extended by many others. ${ }^{3-5}$ In common with broader use of Fourier concepts in physics, the essence of Fourier optics is the description of light diffracted by an object as a sum of spatial sinusoidal waves at specific spatial frequencies. This approach both introduces the concept of spatial frequency and quantifies the imaging properties of an optical systems via an Optical Transfer Function, describing how each spatial frequency is transmitted by an optical system with modified amplitude and phase to form an image.

The detailed mathematical formulation and definitions relating to the Optical Transfer Function are fully described in a number of standard references, ${ }^{4,6}$ but we restrict our study here to the modulus of the Optical Transfer Function known as the Modulation Transfer Function (MTF). It is the MTF that is relevant to understanding imaging using incoherent light.

In order to focus on the key concepts of Fourier optics, the experimental imaging system used consists of a single positive lens and a diffracting aperture (pupil) that image a test pattern (object) from a Spatial Light Modulator (SLM) onto an inexpensive CCD camera. The use of the SLM enables the use of a range of tests targets which can be easily configured (e.g., in amplitude and spatial frequency), and Fourier analysis of the recorded images rapidly gives the intensity of each transmitted frequency and consequently the MTF. The experimental transfer function leads to cut-off frequencies linked to the size and geometry of the pupil used, and we use three different pupils in our experiments. Comparison between experimental results and the theory of Fourier optics provides students with an opportunity to enhance their understanding of Fourier optics and spatial filtering in a general imaging context. 


\section{CONTEXT AND PEDAGOGICAL ISSUES}

Imaging techniques are ubiquitous in industry, research and engineering, and are an essential part of modern courses in physics and engineering. The resolution of an imaging system is commonly specified in terms of its MTF, but this function is often confusing for undergraduate students, often because the observation and measurement of the MTF is not proposed during laboratory classes. Indeed, common experiments on Fourier optics limit laboratory work only to basic ideas relating to the Fourier Transform (FT) such as FT spectrometry, ${ }^{7}$ Fraunhofer diffraction patterns measured over long distances ${ }^{8,9}$ or descreening or spatial filtering in the Fourier plane. ${ }^{10}$

In contrast, we have designed an experiment using only relatively simple and inexpensive equipment that directly yields the MTF and associated autocorrelation function, allowing students to rapidly learn these advanced imaging concepts in parallel with their lecture material. The experiment provides experience in a number of different areas: geometric optics (thin lens equation, magnification); physical optics (polarization, birefringence, phase); optical instrumentation (SLM, CCD camera, an optical bench); and numerical processing (image encoding, Fast Fourier Transform). By combining so many different topics within one laboratory experiment, this also addresses a current problem encountered by many students in physics relating to compartmentalization of knowledge and skills.

\section{OPTICAL TRANSFER FUNCTION}

There are a large number of techniques for measuring the MTF and there is a vast literature dealing with measuring spatial resolution. ${ }^{11}$ One indirect method uses wavefront analyzers to measure the Point Spread Function (PSF) of an optical system, and since the optical transfer function is the Fourier transform of the PSF, it is possible to obtain the modulation transfer function in this way. In contrast, a direct method consists of using test patterns composed of alternative black and white stripes at different spatial frequencies (e.g., USAF 1951 test target) and to measure the resulting contrast in the image. Amongst methods described in the literature, some of the proposed techniques are highly specialized ${ }^{12}$ or outdated ${ }^{13}$ due to the progress in cameras, SLM and computing technology. More recent works use the "knife technique" 14 or the image of a thin slit to get a large and even spatial 
frequency distribution in order to determine the MTF of a camera. ${ }^{15}$

Our experiment extends these existing techniques by replacing the fixed resolution target by a sinusoidal grating with a vertically varying frequency. The grating is displayed on the screen of a SLM and its image is then formed through a positive lens and pupil, before being recorded on a CCD camera. The SLM adds significant versatility to our technique compared to the other methods already proposed. The spatial frequencies and their relative amplitude are precisely defined, and the imaging of a sinusoidal target displayed on the SLM screen gives the students a direct view of the spatial frequency filtering characteristics of the imaging system. And contrary to the methods using test targets, the calculation of the MTF is straightforward. Moreover, the familiarity gained with using an SLM serves an important teaching objective: SLMs are a widespread technology in many domains such as data storage, image and optical signal processing, ${ }^{16}$ optical tweezers, ${ }^{17,18}$ and digital holography, ${ }^{19,20}$ and their use in undergraduate experiments has already been described. ${ }^{21,22}$

\section{A. Optical system under study}

Before describing the experimental setup in detail, we first review the basic filtering properties of the generic imaging system shown in Fig. 1.

A transmitting object is illuminated by an on-axis plane wave $\Sigma$ (coherent or incoherent depending on the source). The object and image planes are conjugate via the thin lens whose useful diameter is limited by the pupil. The transverse coordinates of a point in the object space is $\mathbf{r}(x, y)$ and $\mathbf{r}^{\prime}\left(x^{\prime}, y^{\prime}\right)$ in the image plane. The transverse magnification is $M=\frac{\overline{A^{\prime} B^{\prime}}}{\overline{A B}}=\frac{\overline{O A^{\prime}}}{\overline{O A}}=\frac{X^{\prime}}{X}$. In addition, the pupil is defined by the function $P(x, y): P(x, y)=1$ inside the pupil and $P(x, y)=0$ otherwise. Its FT is denoted $\tilde{P}(u, v)$.

This simple optical system can be analyzed either in the direct space (geometric optics via ray plots) or in the (spatial) frequency space and both spaces are linked by a Fourier Transform. In the frequency space, the effect of the imaging system is that of a low-pass filter characterized by its transfer function. We now describe this transfer function under the particular conditions of incoherent illumination used in our experiments. 


\section{B. Fourier analysis}

A spatial frequency light intensity distribution $\tilde{\mathcal{I}}(\boldsymbol{\nu})$ is associated with a spatial intensity distribution $\mathcal{I}(\mathbf{r})$ via a two-dimensional FT by the usual equation :

$$
\tilde{\mathcal{I}}(\boldsymbol{\nu})=\iint \mathcal{I}(\mathbf{r}) e^{-i 2 \pi \boldsymbol{\nu} \cdot \mathbf{r}} d \mathbf{r}
$$

with $\boldsymbol{\nu}$ the frequency pair $(u, v)$ associated with $\mathbf{r}(x, y)$. In addition to the fact that this is a two dimensional FT, another difficulty of Fourier optics is to know how to express the filtering function $(\mathrm{MTF}=|\mathrm{OTF}|$, see below) in the appropriate frequency space. Indeed, in an imaging system there are two possible spaces: the object space or the image space, with the two being linked by the transverse magnification $M$ (see Fig. 2).

Transfer functions can also be expressed in these two frequency spaces and care should be taken to use the correct variables. In the frequency object space, the transfer functions will be defined via the variable $\boldsymbol{\nu}=\mathbf{r} / \lambda X$ while in the frequency image space they will be a function of the variable $\boldsymbol{\nu}^{\prime}=\mathbf{r}^{\prime} / \lambda X^{\prime}$. Working in the frequency space rather than the direct space is useful because the filtering effects of the imaging system translates into a simple product between its transfer function and the object spatial frequency spectrum (Fig. 2).

\section{Transfer function for incoherent illumination}

In the case of incoherent illumination, the filtering effect is carried out on the distribution of the intensity of the object and the filtering function depends on the lens pupil geometry.

The intensity distribution of the object is $\mathcal{I}_{o b j}(\mathbf{r})$ and that of the image $\mathcal{I}_{\text {ima }}\left(\mathbf{r}^{\prime}\right)$. For incoherent light, these two distributions are linked by a relationship of the type: ${ }^{5}$

$$
\mathcal{I}_{i m a}\left(\mathbf{r}^{\prime}\right) \propto \iint \mathcal{I}_{o b j}(\mathbf{r})\left|\tilde{P}\left(\mathbf{r}^{\prime} / \lambda X^{\prime}-\mathbf{r} / \lambda X\right)\right|^{2} d \mathbf{r}
$$

The filtering function is then given by $|\tilde{P}|^{2}$ (i.e., the squared modulus of the FT of the pupil function) and is called the incoherent Point Spread Function (PSF). Expression (2) is a convolution and it appears in a simpler way in the frequency domain:

$$
\tilde{\mathcal{I}}_{i m a}(\boldsymbol{\nu}) \propto \tilde{\mathcal{I}}_{\text {obj }}(M \boldsymbol{\nu}) \times \operatorname{ITF}(\boldsymbol{\nu})
$$


where ITF stands for Intensity Transfer Function. It is the FT of the PSF and can be expressed as follows:

$$
\operatorname{ITF}(\boldsymbol{\nu})=M \iint P(\mathbf{r}) P^{*}\left(\mathbf{r}+\lambda X^{\prime} \nu\right) d \mathbf{r}
$$

The ITF is the autocorrelation of the pupil function $P(\mathbf{r})$. Its measurement makes it possible to know how an optical system filters the spatial frequencies present in an image. Often we are satisfied with the Modulation Transfer Function (MTF) which is a standardized function and is expressed as:

$$
\operatorname{MTF}(\boldsymbol{\nu})=\left|\frac{\operatorname{ITF}(\boldsymbol{\nu})}{\operatorname{ITF}(\mathbf{0})}\right|=|\operatorname{OTF}(\boldsymbol{\nu})|
$$

The MTF corresponds to the modulus of the Optical Transfer Function (OTF).

\section{EXPERIMENTS IN THE INCOHERENT CASE}

\section{A. Description of the experimental set-up}

The experimental set-up is shown in Fig. 3. The light source is a powerful (up to $700 \mathrm{~mW}$ ) red LED $(\lambda \approx 625 \mathrm{~nm}$ with a Full Width at Half Maximum FWHM $\approx 18 \mathrm{~nm}$ ). The set-up also includes a $0.5 \mathrm{~cm}$ diameter diaphragm limiting the size of the LED emission area (for spatial coherence) and a positive lens $L_{1}$ (actually a $25 \mathrm{~mm}$ diameter collimator with a focal length of $160 \mathrm{~mm}$ ), whose focal point is located on the diaphragm. All these elements provide a wide and parallel beam of incoherent light. This beam is directed by means of a plane mirror towards a first polarizer $\left(P_{1}\right)$ in order to have a linear polarization. A beamsplitter cube (BC) then reflects $50 \%$ of the light back to a low-cost Spatial Light Modulator from Cambridge Correlators (twisted nematic LCoS SLM, model number SDE1024). The resolution of the screen is $768 \times 1024$ with a $9 \times 9 \mu \mathrm{m}$ pixel size, and a VGA interface can be connected to a laptop to use it as a second display such that the display on the SLM can be controlled via software (we used MATLAB ${ }^{\circledR}$ ). The SLM can delay the incident light according to the voltage applied to each pixel with a maximum phase retardation about $0.8 \pi$ at $633 \mathrm{~nm}$. This delay leads to an elliptical polarization of the light reflected back through the $\mathrm{BC}$ and a second polarizer $P_{2}$. The ellipticity and the axes orientation of the elliptical polarization depending on the voltage applied to each pixel, polarizer $P_{2}$ ensures the intensity modulation of the light reflected by each pixel and impinging on the rest of the system. 
Finally, the lens $L_{2}$ (focal length $f^{\prime}=100 \mathrm{~mm}, 1$ " diameter) forms the image of the SLM (in the object plane) on a CCD sensor (in the image plane). An additional pupil is positioned close to $L_{2}$ to form the imaging system under study. The f-number of lens $L_{2}$ is $N \approx 4$ and the proposed technique can be implemented for fast objectives with $N \approx 1$ provided the aberrations are not too important. However, Fourier optics is limited to paraxial optical systems, so it is not suitable for large numerical aperture $(N \leq 1)$. The theory can still be extended with a vector analysis provided the system is aplanatic, but the analysis is much more complex and is beyond the simple discover of Fourier optics for undergraduate students.

The SLM is used to sinusoidally modulate the intensity along one direction (here the modulation will be horizontal). Sinusoidal gratings have the advantage of having only one spatial frequency per horizontal line and therefore allow us to measure how a particular frequency is affected by the pupil-lens system. The SLM has 1024 horizontal pixels along 9.3 $\mathrm{mm}$. Considering the Nyquist-Shannon sampling theorem which states that the maximum frequency contained in the signal must be smaller than half of the sampling frequency (i.e., two pixels per period are needed), the highest frequency of the sinusoidal test pattern that can be displayed is $55.05 \mathrm{~mm}^{-1}$. On the image side, the camera has 1280 (horizontal) pixels with a pitch of $5.2 \mu \mathrm{m}$ so we cannot see spatial frequencies larger than $96.2 \mathrm{~mm}^{-1}$ without aliasing. As a conclusion, in order to measure the specifications of our imaging system (lens and pupil), the system must have a frequency cut-off lower than the frequency limit fixed either by the camera or the SLM depending on the magnification. We also note that variations in the amplitude of the modulation and also in its phase (i.e., its position in the image) can occur due to the imaging system. We will focus on the first one to determine the MTF of $L_{2}$ and its pupil.

Let's add we can neglect in our experiments the effects of the phase modulation due to the SLM and associated with the intensity modulation. Indeed, in our set-up, the spatial coherence radius in the SLM plane is around $0.61 \lambda / \alpha \approx 20 \mu \mathrm{m}$ (with $\alpha$ the maximum incidence angle of the plane waves coming from the source), i.e., 2 SLM pixels. The spatial coherence of the incident field can then modify the MTF for spatial frequencies larger than $50 \mathrm{~mm}^{-1}$ in the object plane, or $100 \mathrm{~mm}^{-1}$ in the image plane (for $|M| \approx 0.5$ ). In the experiments carried out, the frequencies are always below this limit. 


\section{B. Set-up characterization}

Two important parameters of the set-up must be defined before any measurement: the magnification $M$ of the imaging system and the non-linear response of the SLM. Indeed, the intensity modulation achieved by the SLM does not linearly depend on the grey levels of the displayed object and this will affect our study if we do not take it into account. Figure 4 shows the grayscale / intensity experimental response of the SLM. The reflected intensity can be corrected between gray levels 100 and 255 and our sinusoidal grating displayed on the SLM must have an amplitude modulation between theses two values.

Secondly, we need the magnification $M$ of the imaging system SLM- $\left(L_{2}+\right.$ pupil $)-$ CCD before measuring the MTF. It can easily be deduced by displaying on the SLM, e.g., 20 horizontal sinusoidal gratings with frequency varying from 0 to $20 \mathrm{~mm}^{-1}$ and recording the image on the camera through lens $L_{2}$ without any pupils (the pixel size must naturally be taken into account). The images displayed on the SLM screen and recorded by the camera are shown in Fig. 5 .

A FT of the images in Fig. 5 gives the linear relation between frequencies in the object and image planes $\boldsymbol{\nu}=M \boldsymbol{\nu}^{\prime}$ to determine the imaging system magnification $M$ (see Fig. 6). With $M=-0.483$, the object maximum frequency we will be able to see is around $46 \mathrm{~mm}^{-1}$ which is lower than the maximum frequency the SLM can display. Note that the students can check the magnification value with a direct measurement of $X$ and $X^{\prime}$ (see Fig. 3).

\section{Digital processing of the recorded images}

In order to obtain the MTF of the imaging system, two images are needed: the image (through $L_{2}$ and the pupil) of a constant background (gray level 255) displayed on the SLM in order to correct the non-uniformity of pupil-lighting and an image of the target consisting of horizontal sinusoidal gratings whose frequencies vary vertically (e.g., from 0 to $50 \mathrm{~mm}^{-1}$ with a $0.1 \mathrm{~mm}^{-1}$ step). An example of both pictures is shown in Fig. 7 (a) and (b) with a $2 \mathrm{~mm}$ width vertical slit used as a pupil. The main idea is then to calculate the onedimensional Discrete Fourier transform (or DFT) of the target image (b) normalized to the background image (a). The object spatial frequencies are known (because they are displayed on the SLM screen) and the image frequencies are defined with respect to the camera pixel 
size.

The DFT corresponding to image (a) and (b) in Fig. 7 is plotted in the adjacent Fig. 7(c). In the central and upper part of this figure (i.e., for the smallest frequencies), a triangle clearly appears: it corresponds to the spatial frequencies that are passed through the filter that is $L_{2}$ and its pupil. An important noise exists as well inside the filter bandgap and especially around the null frequency. The signal-to-noise ratio can be greatly enhanced in the final result by multiplying the logarithm of the horizontal DFT of the normalized image (image $7(\mathrm{c})$ ) by a binary mask. This mask is null outside two strips defined by the condition $\left|\nu-M \nu^{\prime}\right|<w\left(w=0.5 \mathrm{~mm}^{-1}\right.$ in our case $)$ and equals to 1 inside. Finally, the sum of the elements in each column is needed to get the MTF strictly speaking. The results are outlined in the following subsections.

\section{Experimental results}

1. Slit:

The first pupil we use for $L_{2}$ is an $a=2.00 \pm 0.05 \mathrm{~mm}$ wide vertical slit. The measured magnification is $M=-0.483$ associated with an image-lens plane distance of $X^{\prime}=f^{\prime}(1-$ $M)=14.83 \mathrm{~cm}$. The target displayed on the SLM is a horizontal sinusoidal grating with frequency varying from 0 to $50 \mathrm{~mm}^{-1}$ with a $0.1 \mathrm{~mm}^{-1}$ step. The highest frequencies will be filtered by the camera, but it is not a problem because the optical system cut-off is expected to be lower than $46 / M \approx 95 \mathrm{~mm}^{-1}$.

The autocorrelation of a rectangular function is a triangular function whose cut-off frequency is linked to the slit width $a$. As expected, the measured MTF is triangular-shaped (see solid blue curve in Fig. 8). A non-linear least squares curve fitting with a symmetric triangle function (red dotted line in Fig. 8) gives a cut-off frequency $\nu_{c}= \pm 21.83 \mathrm{~mm}^{-1}$ with a $95 \%$ confidence interval $[21.50,22.16]$ in $\mathrm{mm}^{-1}$ (coefficient of determination $R^{2}=0.9597$ ). This cut-off value is associated to a slit width of $a=\lambda X^{\prime} \nu_{c}=2.04 \pm 0.03 \mathrm{~mm}$ which is in good agreement with the real width. One can note the large amplitude of the null frequency corresponding to the ambient noise (camera, stray light, $\cdots$ ). 


\section{Double slit:}

The slit is replaced by a vertical double slit (width $a=1 \pm 0.05 \mathrm{~mm}$ and space $b=$ $5 \pm 0.05 \mathrm{~mm})$ and the measurements are repeated in the same conditions $(M=-0.483$, $X^{\prime}=14.83 \mathrm{~cm}$ and sinusoidal grating with frequency varying from 0 to $50 \mathrm{~mm}^{-1}$ ). The new transfer function is plotted in Fig. 9 (the null frequency has been removed). It consists of three triangles in accordance with the autocorrelation of two rectangular functions. Because of the particular shape of the MTF, gratings with a high frequency (between $\nu_{c 2}$ and $\nu_{c 3}$ ) clearly appear in the recorded image whereas gratings with smaller frequency (between $\nu_{c 1}$ and $\left.\nu_{c 2}\right)$ are counter-intuitively blurred. Indeed, the students might mistakenly think that, if small details are present in the image, then larger details are also visible in a systematic way. We have here a direct and visual demonstration of the pupil filtering effects and it can provide an improved understanding for the students.

A non-linear least-squares curve fitting with 3 triangle functions can estimate the values of the different frequencies $\pm \nu_{c 1}, \pm \nu_{c 2}, \pm \nu_{c 3}$ and $\pm \nu_{\max }$ (see Fig. 9). The obtained values for the symmetrical frequencies $+\nu_{c i}$ and $-\nu_{c i}(i=1,2)$ and $+\nu_{\max }$ and $-\nu_{\max }$ are equal to the nearest one-thousandth of $\mathrm{mm}^{-1}$. We fix for the fitting $\nu_{c 3}=2 \nu_{\max }-\nu_{c 2}=(a+b) / \lambda X^{\prime}$ and the numerical results are summarized in Table I. From these values we can deduce the geometrical parameters of the pupil $a=1.07 \pm 0.02 \mathrm{~mm}$ and $b=5.00 \pm 0.01 \mathrm{~mm}$, which closely correspond to the theoretical dimensions.

Moreover, the relative amplitude of the small and large triangles are supposed to be in the ratio $2: 1$ if the filtering is solely due to the double slit. However, the heights of the small triangles are 0.35 in Fig. 9 instead of 0.5. This reduction in amplitude is actually due to the MTF of the imaging lens $L_{2}$ (see green dotted line in Fig. 9). The lens has a 1 inch diameter and its MTF is a Chinese hat (chat) function ${ }^{23}$ with a cut-off around $270 \mathrm{~mm}^{-1}$ (cf. Eq. (6)). This frequency is well beyond the cut-off due to the double slit. However, at $\nu_{\max }= \pm 53 \mathrm{~mm}^{-1}$ (peak of the small triangles), the attenuation due to the lens MTF can be theoretically estimated at 0.68 which gives a theoretical height of $0.68 \times 0.5=0.35$ for the small triangles, which exactly corresponds to the measurements. In conclusion the smallerthan-expected height of the lateral triangles is clearly due to the filtering of the imaging lens. 


\section{Circular pupil:}

The last example we show is a pupil of diameter $D$. We first focus on the MTF of a circular aperture (i.e., its autocorrelation). Its mathematical expression is given by: ${ }^{5}$

$$
\operatorname{chat}(u, v)=\frac{2}{\pi}\left[\arccos \left(\frac{\lambda \nu X^{\prime}}{D}\right)-\frac{\lambda \nu X^{\prime}}{D} \sqrt{1-\left(\frac{\lambda \nu X^{\prime}}{D}\right)^{2}}\right]
$$

with $\nu=|\boldsymbol{\nu}|=\sqrt{u^{2}+v^{2}}, u$ and $v$ being the frequencies associated to $x$ and $y$. This chat function only depends on the modulus of its argument and consequently has rotational symmetry. Its graphical representation shown in Fig. 10 resembles a Chinese hat (hence its name) and its cut-off frequency is $\nu_{c}=\frac{D}{\lambda X^{\prime}}$ : any frequency beyond $\nu_{c}$ will not be transmitted between the object and the image.

Measurements with the circular aperture have been performed with a $D=3 \mathrm{~mm}$ diameter pupil and $M$ still equal to -0.483 . The experimental MTF is plotted in Fig. 11 (solid blue line) and it looks like the Chinese hat in Fig. 10. The cut-off frequency is not obvious because of the curvature but it can be estimated with a non-linear least-squares curve fitting according to Eq. 6 (see dashed red line in Fig. 11). The fitted curve accurately describes the experimental data with a coefficient of determination $R^{2}=0.974$ and a cut-off frequency of $\nu_{c}=32.89 \mathrm{~mm}^{-1}$ with a $95 \%$ confidence interval [32.42, 33.35] (in $\mathrm{mm}^{-1}$ ). These last values lead to a pupil diameter of $D=\lambda X^{\prime} \nu_{c}=3.09 \pm 0.04 \mathrm{~mm}$ showing once again good agreement with the real pupil diameter.

\section{CONCLUSION}

We have described here an undergraduate experiment to introduce the essential ideas of Fourier optics. After having tested this experimental work with approximately 40 third year university students in physics, the key points of learning appear to be a clear understanding of the concepts of optical transfer function and spatial frequency, coupled with experimental knowledge of how to determine spatial frequency from a distance measured in the image/object plane. As we have shown, the measured MTF shapes are in good agreement with theory, and this agreement confirms to students the importance of Fourier analysis in optics and spatial filtering. 
Somewhat unexpectedly, the main difficulties encountered were basic optical alignment and the programming required for the image processing. A solution to the first problem is to approximately pre-align the experimental set-up before the laboratory session so that students can save time to correctly place the imaging lens and the camera. A means to address the second problem is to give a written homework question ahead of time on the analysis of the system that requires similar programming in its solution.

This work can be extended to the color response of the optical imaging system ${ }^{15}$ by simply replacing the red LED with a green or blue LED. At a higher level for post-graduate students, aberrations or defective focusing can be also taken into account. For example spherical aberrations or astigmatism disrupt the wavefront after the pupil and reduce the quality of the images leading to a distorted (and degraded) MTF of the optical system solely limited by diffraction. ${ }^{24-26}$ Thus, aberrations may lead to a dramatic drop of the MTF amplitude close to zero and consequently to a large contrast decrease in the images (whereas the theoretical cut-off frequencies remain unchanged).

Overall, this laboratory work is invaluable for students in that it combines physical concepts, advanced instrumentation and numerical processing, and it highlights the use of complex analysis in a practical application in physics.

\section{ACKNOWLEDGMENTS}

This work has been supported by the EIPHI Graduate school (contract "ANR-17-EURE0002"). The authors would like to thank John Dudley for fruitful discussions.

* FEMTO-ST, Université Bourgogne Franche-Comté, 15B avenue des Montboucons, 25030 Besançon cedex; jerome.salvi@univ-fcomte.fr

1 P. M. Duffieux, L'intégrale de Fourier et ses applications à l'optique (Oberthur, Rennes, 1946).

2 P. M. Duffieux, The Fourier Transform and Its Applications to Optics (Wiley, New York, 1983).

3 H. H. Hopkins, Wave Theory of Aberrations (Clarendon Press, 1950).

${ }^{4}$ G. D. Boreman, Modulation Transfer Function in Optical and Electro-Optical Systems, vol. 4 (SPIE press Bellingham, WA, 2001).

5 J. W. Goodman, Introduction to Fourier Optics (Roberts and Company Publishers, 2005). 
6 ISO 9334, "Optics and photonics - Optical transfer function - Definitions and mathematical relationships," Standard, International Organization for Standardization, Geneva (CH) (2012).

7 D. K. Berkey and A. L. King, "An undergraduate experiment in Fourier-transform spectrometry," Am. J. Phys., 40 (2), 267-270 (1972).

8 K. Gan and A. Law, "Measuring slit width and separation in a diffraction experiment," Eur. J. Phys., 30 (6), 1271 (2009).

9 N. G. Ceffa, M. Collini, L. D'Alfonso and G. Chirico, "Hands-on fourier analysis by means of far-field diffraction," Eur. J. Phys., 37 (6), 065701 (2016).

10 S. Ganci, "A simple experiment in non-coherent image processing," Eur. J. Phys., 10 (1), 14 (1989).

11 K. Murata, "Instruments for the measuring of optical transfer functions," in Progress in optics (Elsevier, 1966), vol. 5, pp. 199-245.

12 H. Fujita, D. Y. Tsai, T. Itoh, K. Doi, J. Morishita, K. Ueda, and A. Ohtsuka, "A simple method for determining the modulation transfer function in digital radiography," IEEE Trans. Med. Imaging, 11 (1), 34-39 (1992).

13 D. C. O'Shea and A. C. Rakes, "A modulation transfer function analyzer based on a microcomputer and dynamic ram chip camera," Am. J. Phys., 54 (9), 821-824 (1986).

14 A. P. Tzannes and J. M. Mooney, "Measurement of the modulation transfer function of infrared cameras," Opt. Eng., 34 (6), 1808-1817 (1995).

15 C. Leung and T. D. Donnelly, "Measuring the spatial resolution of an optical system in an undergraduate optics laboratory," Am. J. Phys., 85 (6), 429-438 (2017).

16 D. S. Monaghan, U. Gopinathan, B. M. Hennelly, D. P. Kelly, T. J. Naughton, and J. T. Sheridan, "Applications of spatial light modulators in optical signal processing systems," in Opto-Ireland 2005: Photonic Engineering, edited by T. J. Glynn, G. M. O'Connor, A. J. Flanagan, G. Byrne, J. Magee, J. T. Sheridan, R. F. O'Dowd, G. D. O'Sullivan, and B. W. Bowe, International Society for Optics and Photonics (SPIE, 2005), vol. 5827, pp. 358-368.

17 M. Reicherter, T. Haist, E. Wagemann, and H. J. Tiziani, "Optical particle trapping with computer-generated holograms written on a liquid-crystal display," Opt. Lett., 24 (9), 608-610 (1999).

18 J. Liesener, M. Reicherter, T. Haist, and H. J. Tiziani, "Multi-functional optical tweezers using computer-generated holograms," Opt. Commun., 185 (1-3), 77-82 (2000). 
19 T. Kreis, Handbook of Holographic Interferometry: Optical and Digital Methods (John Wiley \& Sons, 2006).

20 T.-C. Poon and J.-P. Liu, Introduction to Modern Digital Holography: with MATLAB (Cambridge University Press, 2014).

21 M. Jacquot, O. Iken, B. Bobillier, S. Cremer, L. Froehly, F. Courvoisier, L. Furfaro, R. Giust, and J. Dudley, "Montage d'holographie numérique dynamique pour la nano-métrologie optique sans lentille," J3eA, 14, 1006 (2015).

22 D. Huang, H. Timmers, A. Roberts, N. Shivaram, and A. S. Sandhu, "A low-cost spatial light modulator for use in undergraduate and graduate optics labs," Am. J. Phys., 80 (3), 211-215 (2012).

23 R. Bracewell, Fourier Analysis and Imaging (Springer Science \& Business Media, 2004).

24 H. H. Hopkins, "The frequency response of a defocused optical system," Proc. R. Soc. Lond. A Mat., 231 (1184), 91-103 (1955).

25 C. S. Williams and O. A. Becklund, Introduction to the Optical Transfer Function (Wiley, 1989).

26 M. Born and E. Wolf, Principles of Optics: Electromagnetic Theory of Propagation, Interference and Diffraction of Light (Elsevier, 2013), pp. 547-553.

TABLE I. Numerical results of the non-linear least-squares fitting of the experimental curve in Fig. 9. The coefficient of determination is $R^{2}=0.983$.

\begin{tabular}{cccc}
\hline \hline Frequency & Fitted value & $95 \%$ confidence bounds & Theoretical frequency \\
\hline$\nu_{c 1}$ & $11.37 \mathrm{~mm}^{-1}$ & {$[11.14,11.60]\left(\mathrm{mm}^{-1}\right)$} & $\frac{a}{\lambda X^{\prime}}$ \\
$\nu_{c 2}$ & $43.18 \mathrm{~mm}^{-1}$ & {$[43.01,43.35]\left(\mathrm{mm}^{-1}\right)$} & $\frac{a-b}{\lambda X^{\prime}}$ \\
$\nu_{c 3}$ & $63.4 \mathrm{~mm}^{-1}$ & {$[63.1,63.7]\left(\mathrm{mm}^{-1}\right)$} & $\frac{a+b}{\lambda X^{\prime}}$ \\
$\nu_{\max }$ & $53.30 \mathrm{~mm}^{-1}$ & {$[53.23,53.37]\left(\mathrm{mm}^{-1}\right)$} & $\frac{b}{\lambda X^{\prime}}$ \\
\hline \hline
\end{tabular}




\section{LIST OF FIGURES}

1 Image formation scheme with a positive thin lens. With the sign convention used, the image distance $X^{\prime}$ is positive (the image is real) and the object distance $X$ is negative. The inverted image corresponds to a negative transverse

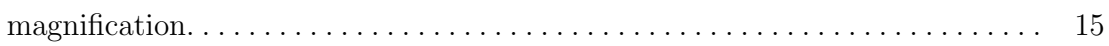

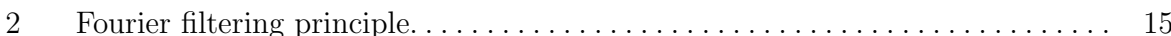

3 Scheme of the experimental set-up to measure the pupil filtering effects with incoherent illumination (see text for details). . . . . . . . . . . . . 15

4 Measured intensity response of the SLM versus the gray level displayed. The non-linearity can be corrected between 100 and $255 \ldots \ldots \ldots \ldots \ldots \ldots \ldots$

5 Images used to calculate the magnification $(M<0)$. Left: sinusoidal gratings displayed on the SLM screen with a frequency varying from 0 to $20 \mathrm{~mm}^{-1}$ (with compensated intensity non-linearity). Right: corresponding Region Of Interest $(\mathrm{ROI})$ recorded on the camera $(1280 \times 1024$ pixels and $5.2 \mu \mathrm{m}$ pixel pitch).

6 Spatial frequency in the image versus the corresponding frequency in the object. The slope of the line is $1 /|M| \ldots \ldots \ldots \ldots \ldots \ldots \ldots \ldots \ldots \ldots \ldots$

7 Preliminary results with a $2 \mathrm{~mm}$ vertical slit. (a): Background image; (b): Image of the target; (c): Logarithm of the horizontal DFT of the normalized image (ratio between images (b) and (a) . . . . . . . . . . . . . 15

8 Solid blue line: normalized experimental MTF with an $a=2 \mathrm{~mm}$ slit ( $M=$ -0.483). Dashed red line: fitted curve. . . . . . . . . . . . . . . . 15

9 Solid blue line: experimental MTF with two slits $a=1 \mathrm{~mm}$ wide and $b=5$ mm apart. Dashed red line: fitted curve. Dotted green line: theoretical MTF of imaging lens $L_{2}$ (chat function defined by Eq. (??) with a 1" diameter). . . 15

10 (a): 2D theoretical MTF chat of a circular pupil with diameter $D$. (b): crosssection along $u$ direction. Its frequency cut-off is $\nu_{c}=\frac{D}{\lambda X^{\prime}} \ldots \ldots \ldots \ldots \ldots$

11 Experimental MTF (solid blue line) with a $D=3 \mathrm{~mm}$ diameter circular pupil and corresponding fitted curve with theoretical MTF from Eq. ?? (red dashed

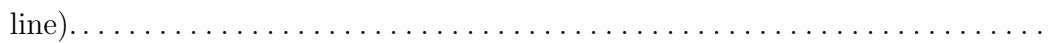


FIG. 1. Image formation scheme with a positive thin lens. With the sign convention used, the image distance $X^{\prime}$ is positive (the image is real) and the object distance $X$ is negative. The inverted image corresponds to a negative transverse magnification.

FIG. 2. Fourier filtering principle.

FIG. 3. Scheme of the experimental set-up to measure the pupil filtering effects with incoherent illumination (see text for details).

FIG. 4. Measured intensity response of the SLM versus the gray level displayed. The non-linearity can be corrected between 100 and 255 .

FIG. 5. Images used to calculate the magnification $(M<0)$. Left: sinusoidal gratings displayed on the SLM screen with a frequency varying from 0 to $20 \mathrm{~mm}^{-1}$ (with compensated intensity non-linearity). Right: corresponding Region Of Interest (ROI) recorded on the camera $(1280 \times$ 1024 pixels and $5.2 \mu \mathrm{m}$ pixel pitch).

FIG. 6. Spatial frequency in the image versus the corresponding frequency in the object. The slope of the line is $1 /|M|$.

FIG. 7. Preliminary results with a $2 \mathrm{~mm}$ vertical slit. (a): Background image; (b): Image of the target; (c): Logarithm of the horizontal DFT of the normalized image (ratio between images (b) and (a).

FIG. 8. Solid blue line: normalized experimental MTF with an $a=2 \mathrm{~mm}$ slit $(M=-0.483)$. Dashed red line: fitted curve.

FIG. 9. Solid blue line: experimental MTF with two slits $a=1 \mathrm{~mm}$ wide and $b=5 \mathrm{~mm}$ apart. Dashed red line: fitted curve. Dotted green line: theoretical MTF of imaging lens $L_{2}$ (chat function defined by Eq. (6) with a 1" diameter).

FIG. 10. (a): 2D theoretical MTF chat of a circular pupil with diameter D. (b): cross-section along $u$ direction. Its frequency cut-off is $\nu_{c}=\frac{D}{\lambda X^{\prime}}$. 
FIG. 11. Experimental MTF (solid blue line) with a $D=3 \mathrm{~mm}$ diameter circular pupil and corresponding fitted curve with theoretical MTF from Eq. 6 (red dashed line). 